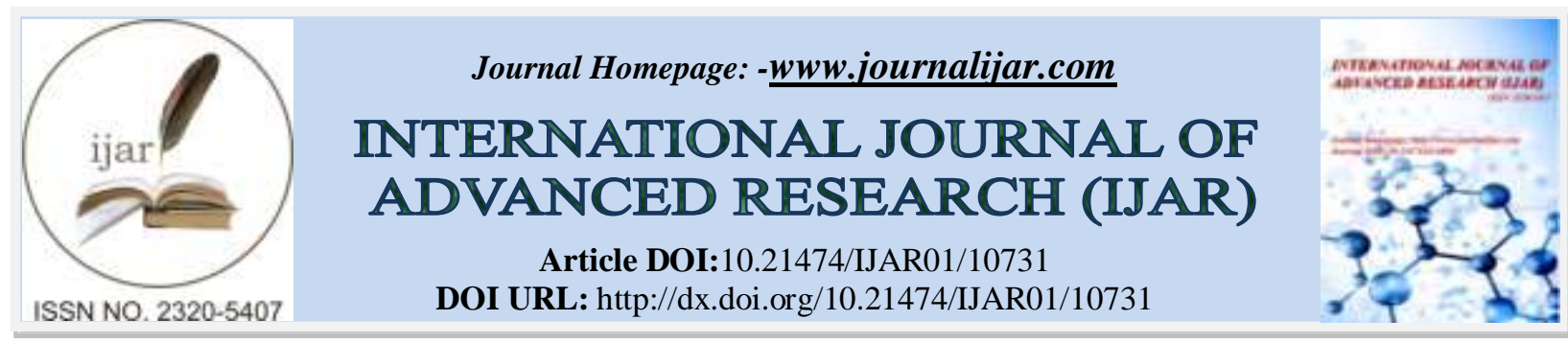

RESEARCH ARTICLE

\title{
COMPARATIVE PHARMACOLOGICAL INVESTIGATIONS OF N-HEXANE FRACTIONS OF BAUHINIA SCANDENS LINN.STEMS AND SENNA SOPHERA L. STEMS
}

\author{
Nazim Uddin Emon ${ }^{1}$, Israt Jahan ${ }^{1}$, Safaet Alam ${ }^{2}$, Md. Mohotasin Hossain ${ }^{1}$, Sheikh Md. Kamrul Hasan ${ }^{3}$, Md. \\ Munsur Alam ${ }^{1}$, Md. Solaman Uddin Sawon ${ }^{1}$, Sajib Rudra ${ }^{4}$, Mohammad Nazmul Islam ${ }^{1}$, and Dr. Mohammed \\ Aktar Sayeed ${ }^{1}$ \\ 1. Department of Pharmacy, International Islamic University Chittagong, Chittagong - 4318, Bangladesh. \\ 2. Department of Pharmaceutical Chemistry, Faculty of Pharmacy, University of Dhaka, Dhaka-1000, Bangladesh. \\ 3. Department of Pharmacy, Stamford University Bangladesh, Dhaka - 1217, Bangladesh. \\ 4. Department of Botany, University of Chittagong, Chittagong - 4331, Bangladesh.
}

\section{Manuscript Info}

Manuscript History

Received: 25 January 2020

Final Accepted: 27 February 2020

Published: March 2020

Key words:-

Bauhinia Scandens, Senna Sophera, Clot

Lysis, Cytotoxicity, Streptokinase

\section{Abstract}

Objectives:Bauhinia scandens Linn,and Senna sopherahas been locally known as monkey ladders and Kalkasunda in Bangladesh.They have several significant traditional uses as a source of the medicinal agent. This study is to implement the thrombolytic and cytotoxic activity of n-Hexane fraction of Bauhinia scandensstems and Senna sopherastems (NHBS, and NHSS).

Methods: In this study, in vitro clot lysis method and brine shrimp lethality method were implemented to check the thrombolytic and cytotoxic potentiality of NHBS and NHSS.

Results: In the thrombolytic test, NHBS $(24.52 \pm 2.86 \%$; $\mathrm{P}<0.001)$ attained a significant percentage of clot lysis but NHSS (16.59 \pm $4.07 \%$; $\mathrm{P}<0.001$ ) showed moderate clot lysis potentiality in human blood. Again, the LD50 value of NHBS and NHSS was seen at 92.55 $\mu \mathrm{g} / \mathrm{mL}$ and $338.54 \mu \mathrm{g} / \mathrm{mL}$ of doses respectively in brine shrimp lethality bioassay.

Conclusion: Present results suggested, NHBS showed comparatively potent thrombolytic activity than NHSS, besides, a comparative study ofthe LD50 value of NHBS and NHSS suggested that the NHBS has strong anti-tumor potentiality other than NHSS.

CopyRight, IJAR, 2020,. All rights reserved.

\section{Introduction:-}

The exceptional phenomena of symbiosis are often exemplified by nature as a golden mark. The treatment of human disease has been centered on natural products including plants, animals, and minerals.In the last decades, demand for plants is constantly growing throughout the globe which is one of the richest sources of promising versatile chemical compounds and medicines. Plants could, therefore, play a large role in exploring new stockpiles for discovering new disease challenges. The therapeutic properties of the plant have been studied because of their strong pharmacological efficacy, low toxicity and low economic viability[1]. In developed countries, thrombosis is a key pathophysiological process underlying acute coronary disturbances that are the major causes of morbidity and death. Portal venous thrombosis often caused by the forming of thrombus in the vein that leads to portal arterial blockage and portal hypertension. Occlusion of the cerebral epithelial duct can be a common disorder, among key 
cardiovascular events. The diagnosis of patients with cerebral epithelial duct occlusion is made possible by clotbuster drugs including proteinase, alteplase (activase), reteplase (Retavase), tenecteplase (TNKasase, Streptokinase, Urokinase, etc[2]. Most thrombolytic regimens that are currently available have some vital deficiencies including minimal fibrin specificity, significant bleeding, and the high dose needs when they are used to lyse existing blood coagulation in clinical configurations in which ischemia (critical myocardial infarction, lung embolism, ischemia, and arterial thrombotic infarction) can be suspicious[3].Choriocarcinoma medication along with chemotherapy is also managed with thrombolytic therapy[4].Cytotoxicity screening models provide valuable preliminary information for selecting plant extracts for future research with possible anti-neoplastic properties [5]. This is why scientists are looking for less injurious human cytotoxic substances. The use of plants like spices and their components as possible chemo-preventive elements persist a comprehensive research subject in the field of preventing cancer. Several studies on the relation of plant use, cancer prevention, antimicrobial consequences, and overall human health protection were published [6].

Throughout the Caesalpinioideae sub-family of the broader Fabaceae family,Bauhinia scandens Linn is a genus of more than 500 species of flowering plants thatis locally called snake-climber or monkey ladder. Many species of these plants have been cultivated in the tropical regions especially in Bangladesh, India, Sri Lanka, Vietnam, and Southeast China Many ethnomedicinal reports and local practitioners have suggested that it is a flowering plant that has numerous medicinal properties that local practitioners use directly for the use of various conditions such as Alzheimer's disease, acute dysentery, heart disease, tumors, etc; [7]. Senna sophera(Family: Fabaceae) also belongs to the Caesalpinioideae subfamily of the Fabaceae class, locally called Kalkasunda or Jhign. It is found in Bangladesh, India and most of the tropical region. Traditionally, all plant parts are used for numerous medicinal purposes. The complete plant is a febrifuge and laxative. The leaves of Senna sophera are used as a diuretic in psoriasis, ringworm, arthritis, cough, skin disease and the heart. This plant has been used by former Indian doctors for respiratory illness[8]. The object and aim of the study are to revel and evaluate the thrombolytic and cytotoxic activities of n-Hexane fraction of B. Scandesstems and S. sophera stems (NHBS, NHSS).

\section{Materials and Methods:- Drugs and Chemicals:}

In this study, Tween 80, DMSO, and n-Hexane were purchased from Sigma Chemicals Co., U.S.A. Lyophilized streptokinase vial (15,00000 IU) was obtained from Square Pharmaceuticals Ltd, Bangladesh, Sodium Chloride $(\mathrm{NaCl})$ was brought from Orion Pharmaceuticals Ltd, and Bangladesh and Vincristine sulfate (1 mg/vial) was purchased from Beacon Pharmaceuticals Ltd, Bangladesh.

\section{Plant materials:}

For this present investigation, plant sample (steams) of B. scandens and S. sophera was collected from Sitapahar, Kaptai, Rangamati, Bangladesh, in September 2018. Then proper identification of plant sample was done by Dr. Shaikh Bokhtear Uddin, Professor, Department of Botany, University of Chittagong, Chittagong-4331, Bangladesh. The specimen voucher (CTGUH SR7918) for Bauhinia Scandens and (CTGUH SR 8391) for Senna sophera were deposited at the Department of Pharmacy, International Islamic University of Chittagong, Chattrogram-4318, Bangladesh for further reference (ref no: Pharm P\&D - 27/38'-19).

\section{Preparation of methanol crude extract and n-hexane fraction:}

After collection of stems, they have been dried for 30 days at $23 \pm 2^{0} \mathrm{C}$ in the natural shade. The plant materials were then dried up the oven for 1 hour $\left(40^{\circ}\right.$ C $)$ for proper grinding at a relatively low temperature. The dried stems were then grounded with a high-speed grinding machine to form coarse powder in a well-closed plastic container the powdered plant material was stored and kept in a cool, dark and ventilated place. Roughly 500 g of the powdered plant material has been extracted and all of them were soaked into two liters of methanol in a separate clean glass container. Containers with their contents have been sealed with aluminum foil with strong lid and kept at $23 \pm 2^{0} \mathrm{C}$ for 14 days, accompanying intermittent shaking and stirring. The whole mixture was filtered via cotton and then the filtrates were condensed in a water bath at $40^{\circ} \mathrm{C}$ with a double ring filter paper. The weight of the crude methanol extract obtained from $B$. scandens stems was $17 \mathrm{~g}$ and $15 \mathrm{~g}$ from $S$. sophera stems. The solution was then thoroughly shaken with $n$-hexane and then the top part was gently removed by the separating funnel. With the help of a water bath, the solvent was completely evaporated and $1.7 \mathrm{~g}$ NHBS and a $1.3 \mathrm{~g}$ NHSS n-hexane fraction were obtained which used for further study. Following the protocol established by Kupchan and developed byWagenen et al. [9] was performed to separate n-Hexanee fraction. 


\section{Thrombolytic activity:}

The clot lysis study was performed according to the described Prasadet;al.[10]method, as a stock solution, lyophilized streptokinase vial (15.00000 I.U.) appropriately mixed with $5 \mathrm{~mL}$ sterile distilled Water through which dilution was made. For each herbal extract (NHBS, NHSS), whole blood was taken from the healthy volunteers ( $\mathrm{n}=$ 10) who didn't take any oral anticoagulants and contraceptives for nearly 10 days. After collection, the blood samples were allowed to from clot in 15 pre-weighed sterile Eppendorf tubes. $0.5 \mathrm{~mL}$ of freshly obtained blood was introduced to each correctly labeled tube and put into clot formation for 45 minutes by incubating at $37^{\circ} \mathrm{C}$.After its formation, the serum was completely removed from each tube and weighed without disturbing clot. For each concentrate tube, $100 \mu \mathrm{L}(100 \mu \mathrm{g} / 10 \mathrm{~mL})$ of the test sample (NHBS and NHSS), the streptokinase (as positive control) and the saline water (negative control) were separately added and were incubated for $90 \mathrm{~min}$ at $37^{\circ} \mathrm{C}$, then removed the clot lysis again, and weighed once more to obtain the weight difference between primary and final weight conducted for clot lysis (thrombolysis). This procedure was then replicated 3 times with the same five participant's blood samples. The level of clot lysis was determined to utilize the accompanying equation:

$\%$ of clot lysis $=($ weight of clot after remove of fluid/clot weight $) \times 100$

\section{Brine shrimp lethality bioassay:}

The method outlined by Meyeret; al.[11] was accompanied to check the cytotoxicity of the n-hexane fraction $B$. scandens and S. sophera stems. Next, the dried extract was dissolved in DMSO (50 $\mu \mathrm{L}$ in $5 \mathrm{~mL}$ solution) to ready the test sample with artificial seawater $(3.8 \% \mathrm{NaCl}$ in water) to achieve the serially diluted concentrations of 31.25 , $62.5,125,250,500$ and $1000 \mu \mathrm{g} / \mathrm{mL}$. A sequence concentration dilution $0.125,0.25,0.5,1,5$ and $10 \mu \mathrm{g} / \mathrm{mL}$ of Vincristine sulfate were used as a positive control of the predecessor process. Shrimp was used for cytotoxicity analysis and effective surveillance. Eggs of Brine shrimps from the nearby aquarium shop (Chittagong, Bangladesh) were obtained and then hatched in artificial seawater under constant light for 24 hours with continuous air supply. Nauplii or Brine shrimp larvae were left to grow in the hatched shrimp for 48 hours. After 48 hours the nauplii were separated from the eggs and put with artificial marinated water in a glass petri dish, they were actively carried to one side of the petri dish.The suspension containing 20 nauplii was poured into each test tube and was incubated at room temperature $\left(25 \pm 10^{\circ} \mathrm{C}\right)$ under light for 24 hours. The test tubes were then assessed after 24 hours and a $3 \mathrm{X}$ magnifying glass used for counting the number of surviving nauplii in each tube. "Microsoft Excel 2013" software was used to determine a linear regression equation for the concentration that would kill 50\% of nauplii (LC50).

\section{Statistical analysis:}

The significance between \% clot lysis by Streptokinase and herbal extract employing weight difference was tested by using Graph Pad Prism version 5.2. Statistical significance was dictated by One Way Analysis of Variance (ANOVA) accomplished by Dunnett's Multiple Comparison test and data are expressed as mean \pm standard deviation. In the case of Brine shrimp cytotoxicity "Microsoft Excel 2013" software was used to determine a linear regression equation for several concentrations.

\section{Results:-}

Thrombolytic activity:

The thrombolytic activity n-hexane extract of B. scandens and S. sophera stem summarized in Figure 1. The showed the highest percentage of clot lysis was seen $24.52 \%$ for NHBS $(P<0.001)$ whereas, NHSS showed $16.59 \%$ of clot lysis activity $(P<0.01)$ in comparison to negative control saline water attained $6.62 \%$ of clot lysis, and the standard drug streptokinase showed $51.31 \%,(P<0.001)$ clot lysis activity. The final orders for the percentage of clot lysis were as followed: streptokinase > NHBS > NHSS > saline water. 


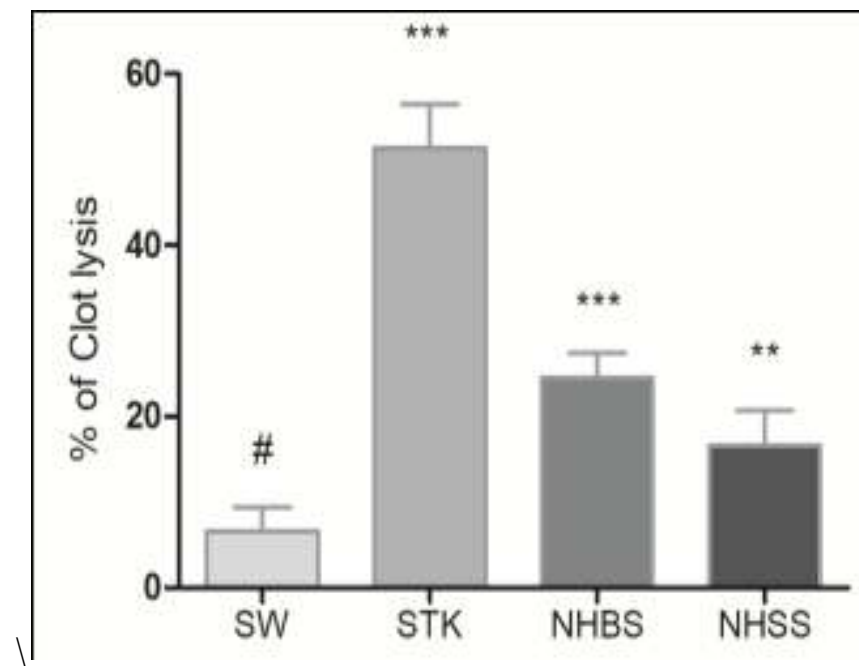

Figure 1:- Clot lysis effects by Saline water, Streptokinase, NHBS, and NHSS. Clot-lysis values are presented as mean \pm SEM ( $\mathrm{n}=10)$; One-way analysis of variance (ANOVA) was followed by Dunnett's test. $* * * P<0.001$ and $* * P<0.01$ were considered as significant compared with the control, where \# is designated as control. NHBS $=\mathrm{n}$ Hexane fraction of $B$. scandens stems, NHSS = n-Hexane fraction of Senna sophera stems, SW = saline water, and STK $=$ streptokinase.

\section{Brine shrimp lethality bioassay:}

In brine shrimp lethality bioassay, the n-hexane fractions of $B$. scandens and $S$. sophera stem exhibited a positive result in comparison with the positive control vincristine sulfate. By plotting the concentration versus the percentage of mortality for all test samples showed an approximately linear correlation. The median lethal concentrations or (LC50, the concentration at which 50\% mortality of brine shrimp nauplii occurred) were determined from the graph. Cytotoxic effects of the samples have been shown in Figure 2. The LC50 for the NHBS was found $92.55 \mu \mathrm{g} / \mathrm{mL}$, with the regression value of $y=0.0637 x+44.102, R^{2}=0.7631$ and NHSS was found $338.54 \mu \mathrm{g} / \mathrm{mL}$ which yielded regression value was found $y=0.0784 x+23.452, R^{2}=0.9875$ respectively, and finally the LD50 was attained 2.16 $\mu \mathrm{g} / \mathrm{mL}$ for standard (Vincristine sulfate) was which regression value $\mathrm{y}=7.6315 \mathrm{x}+33.536, \mathrm{R}^{2}=0.8585$.

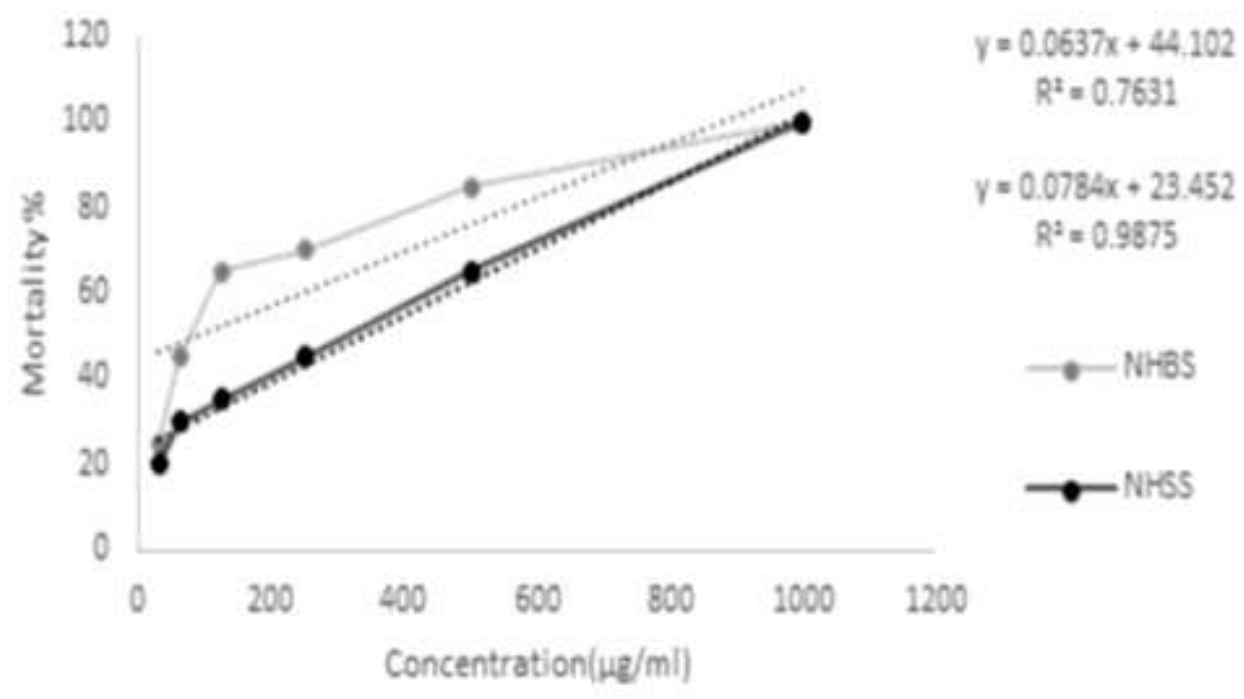

Figure 2: LC50 (median lethal concentration) value of NHBS and NHSS. Where, NHBS $=$ n-Hexane fraction of $B$. scandensstems and NHSS $=\mathrm{n}-H e x a n e$ fraction of $S$. sophera stems. 


\section{Discussion:-}

About $30 \%$ of the medication ingredients globally are made from plants. Several studies were carried out by various scientists to determine the thrombolytic effect (anticoagulants and anti-platelets) of herbs and natural food sources and their supplements. There is proof of the prevention of heart attacks and stroke by the use of these items[12]. There have been several thrombolytic drugs, particularly recombinant DNA therapies, and there are many other associated side effects. This side effect has been reported to lead to further complications[13]. In our present study, two different extracts of two diverse plants showed the thrombolytic activity between which the crude n-hexane fractions of $B$. scandens stem had a significant activity than the $S$. sophera stems. The maximum clot lysis activity was mostly observed in n-hexane fraction of B. scandens stems that mean n-hexane soluble compounds of $B$. scandens stems and $S$. sophera stems contains thrombolytic responsible constituents. Obviously, plants with plasminogen receptor binding plasminogen are infected by the bacterium[14]. The plasminogen cell-binding surface is readily stimulated through plasmin that can result in fibrinolysis whereas other plants are using their enzymes as a fibrinolysis effect[15]. Furthermore, the plasminogen activator bacterial, Staphylokinase streptokinase, serves as cofactor molecules that help to form exosites and enhance the enzyme's substrate presentation. Staphylokinase stimulates plasminogen to dissolve the blood clotting and also destroys cellular extra-cellular matrix and fibrin fibers[16]. Again, the bioassays for brine shrimp lethality are simple and straight forward benchtops for predicting essential activities like enzyme inhibition, ion channel, antimicrobial, and cytotoxic activity. LC50 value is considered non-toxic over $1000 \mu \mathrm{g} / \mathrm{mL}$, weakly toxic from ranging $500-1000 \mu \mathrm{g} / \mathrm{mL}$, moderately toxic to $100-500$ $\mu \mathrm{g} / \mathrm{mL}$, and very toxic for less than $100 \mu \mathrm{g} / \mathrm{mL}$ [17]. In our study $\mathrm{n}$-hexane fraction of $B$. scandens stem extract showed high toxicity $(\mathrm{NHBS}=92.55 \mu \mathrm{g} / \mathrm{mL}$ ) where $\mathrm{n}$-hexane fraction of $S$. sophera stem showed weak toxicity (NHSS $=338.54 \mu \mathrm{g} / \mathrm{mL}$ ) upon compared to LC50 value of Vincristine sulfate. Comparison of this result with the standard Vincristine sulfate $(0.76 \pm 0.04 \mu \mathrm{g} / \mathrm{mL})$ indicated that the lethality of NHBS suggesting thenotable and NHSS suggesting comparatively weak clinical importance of the crude drug against tumor cells, pesticides etc.

\section{Conclusion:-}

The study reported that the n-Hexane fraction of $B$. scandens and $S$. sophera stems has got the significant as a thrombolytic agent. On the other hand, n-Hexane fraction of $B$. scandens stems showed notable toxicity upon compared with S.sophera which very weak toxicity. That's why the n-Hexane fraction of $B$. scandens is strongly recommended as an anticoagulant and as a thriving source of anti-tumor substance. Furthermore, this is only a preliminary study and to make a final comment the sample should be thoroughly investigated by chemically to exploit their medicinal and pharmaceutical potentials.

\section{Ethical Consideration:}

The experimentalprotocol has been approved by the P and D (Pharm P\&D - 27/38-19) committee of the Department of Pharmacy, International Islamic University Chittagong, Chittagong - 4318, Bangladesh.

\section{Competing interest:}

None to declare

\section{Funding:}

Not applicable.

\section{Acknowledgment:-}

The authors would like to have their gratitude to Dr. Shaikh Bokhtear Uddin, Professor, Department of Botany, University of Chittagong, Chittagong - 4331, Bangladesh for the identification of plants. Besides, the authors also like to thanks volunteers and the Department of Pharmacy, International Islamic University Chittagong, Chittagong -4318 , Bangladesh for providing lab facilities.

\section{Reference:-}

1. Rai, P.K., et al., Glycemic Properties of $<\mathrm{i}>$ Trichosanthes dioica $</ \mathrm{i}>$ Leaves. Pharmaceutical Biology, 2008. 46(12): p. 894-899.

2. Bekker, J., S. Ploem, and K.P. De Jong, Early hepatic artery thrombosis after liver transplantation: A systematic review of the incidence, outcome and risk factors. American Journal of Transplantation, 2009. 9(4): p. 746-757. 
3. Sisay, M., E. Engidawork, and W. Shibeshi, Evaluation of the antidiarrheal activity of the leaf extracts of Myrtus communis Linn (Myrtaceae) in mice model. BMC complementary and alternative medicine, 2017. 17(1): p. 103.

4. Yang, M. and L. Peng, Role of chemotherapy and thrombolysis in treatment of choriocarcinoma accompanied with pulmonary embolism. Medicine, 2017. 96(36): p. e7866-e7866.

5. Itharat, A., et al., In vitro cytotoxic activity of Thai medicinal plants used traditionally to treat cancer. Journal of Ethnopharmacology, 2004. 90(1): p. 33-38.

6. Dzoyem, J.P., et al., Cytotoxic and antimicrobial activity of selected Cameroonian edible plants. BMC complementary and alternative medicine, 2013. 13(1): p. 78.

7. Hossain, M.S., et al., Antioxidant Activities and HPLC-DAD Based Phenolic Content Determination of Bauhinia scandens. Journal of Pharmaceutical Research International, 2016: p. 1-9.

8. Emon, N.U., et al., In Vitro Comparative Cardioprotective Activity of Methanol Extract of Caesalpinia Digyna (Rottl.) Stems and Senna Sophera (L.) Roxb. Stems.

9. Mahfuz, A., et al., Characterization of in-vitro antioxidant, cytotoxic, thrombolytic and membrane stabilizing potential of different extracts of Cheilanthes tenuifolia and Stigmasterol isolation from n-hexane extract. Clinical Phytoscience, 2019. 5(1): p. 39.

10. Prasad, S., et al., Development of an in vitro model to study clot lysis activity of thrombolytic drugs. Thrombosis Journal, 2006. 4.

11. Sasidharan, A.T.K. and K.K. Elyas, Anti-fungal Potential and Brine Shrimp Lethality Assay of in vitro Raised Clones of Celastrus paniculatus. Sciences, 2019. 12(4): p. 877-883.

12. Prasad, S., et al., Effect of Fagonia arabica (Dhamasa) on in vitro thrombolysis. BMC Complementary and Alternative Medicine, 2007. 7(1): p. 36.

13. Priestman, T., The Theoretical Basis of Cancer Chemotherapy. 2007, Springer London: London. p. 1-34.

14. Emran, T.B., et al., Effects of organic extracts and their different fractions of five Bangladeshi plants on in vitro thrombolysis. BMC complementary and alternative medicine, 2015. 15(1): p. 128.

15. Emon, N.U., I. Jahan, and M.A. Sayeed, Investigation of antinociceptive, anti-inflammatory and thrombolytic activity of Caesalpinia digyna (Rottl.) leaves by experimental and computational approaches. Advances in Traditional Medicine, 2020: p. 1-9.

16. Parry, M.A., X.C. Zhang, and W. Bode, Molecular mechanisms of plasminogen activation: bacterial cofactors provide clues. Trends in biochemical sciences, 2000. 25(2): p. 53-59.

17. da Costa, J.G., et al., Biological screening of araripe basin medicinal plants using Artemia salina Leach and pathogenic bacteria. Pharmacognosy Magazine, 2010. 6(24): p. 331-331. 